\title{
EMBEDDING INVERSE SEMIGROUPS OF HOMEOMORPHISMS ON CLOSED SUBSETS
}

\author{
by BRIDGET BOS BAIRD
}

\author{
(Received 29 April, 1976)
}

All topological spaces here are assumed to be $T_{2}$. The collection $\mathscr{I}_{F}(Y)$ of all homeomorphisms whose domains and ranges are closed subsets of a topological space $Y$ is an inverse semigroup under the operation of composition. We are interested in the general problem of getting some information about the subsemigroups of $\mathscr{F}_{F}(Y)$ whenever $Y$ is a compact metric space. Here, we specifically look at the problem of determining those spaces $X$ with the property that $\mathscr{I}_{F}(X)$ is isomorphic to a subsemigroup of $\mathscr{I}_{F}(Y)$. The main result states that if $X$ is any first countable space with an uncountable number of points, then the semigroup $\mathscr{I}_{F}(X)$ can be embedded into the semigroup $\mathscr{F}_{F}(Y)$ if and only if either $X$ is compact and $Y$ contains a copy of $X$, or $X$ is noncompact and locally compact and $Y$ contains a copy of the one-point compactification of $X$.

Various corollaries follow quickly. For example the one-point compactification of the Euclidean $n$-space $\mathbb{R}^{n}$ is the Euclidean $n$-sphere $S^{n}$ and, of course, $S^{n}$ can be embedded in $I^{m}$, the compact $m$-dimensional Euclidean cube, if and only if $n<m$. Consequently, an application of the main theorem yields the fact that the semigroup $\mathscr{I}_{F}\left(\mathbb{R}^{n}\right)$ can be embedded in the semigroup $\mathscr{I}_{F}\left(I^{m}\right)$ if and only if $n<m$.

Some of the results which are preliminary to the main result make no requirements on the spaces involved. In particular, they do not require either of the spaces to be compact. We apply these results to get some information about embedding $\mathscr{I}_{F}(X)$ into $\mathscr{I}_{F}(Y)$ when either $X$ or $Y$ is discrete. When $X$ is discrete, $\mathscr{I}_{F}(X)$ is just the symmetric inverse semigroup on the set $X$. Finally, we show that the requirement in the main theorem that $X$ have an uncountable number of points is essential by producing a countable metric space $X$, a compact metric space $Y$ and an isomorphism from $\mathscr{I}_{F}(X)$ into $\mathscr{I}_{F}(Y)$, even though $Y$ contains neither a copy of $X$ nor of the one-point compactification of $X$.

1. Some preliminary discussion. Our notation and terminology will generally conform to that of [1]. We recall that two elements $a$ and $b$ of a semigroup $S$ are inverses of each other if $a b a=a$ and $b a b=b$, and that $S$ is defined to be an inverse semigroup if each element has a unique inverse. The canonical example of an inverse semigroup is the symmetric inverse semigroup $\mathscr{I}_{X}$ which is simply the semigroup under composition of all injective partial functions on a set $X$. This semigroup plays much the same role in the theory of inverse semigroups as the symmetric group plays in the theory of groups and as the full transformation semigroup plays in the theory of semigroups. In particular, every inverse semigroup can be embedded in some symmetric inverse semigroup. This was proven by V. V. Vagner [5] in 1952 and independently by G. B. Preston [4] in 1954.

We consider in this paper the semigroup $\mathscr{I}_{F}(X)$ of all homeomorphisms between closed subsets of $X$; this, as we mentioned previously, coincides with the symmetric inverse semigroup 
on $X$ when $X$ is discrete. For any $f \in \mathscr{I}_{F}(X), \operatorname{dom} f$ and $\operatorname{ran} f$ will denote respectively the domain and range of $f$. For $f$ and $g$ in $\mathscr{I}_{F}(X)$, we define

$$
f \circ g=\{(x, y):(x, z) \in g \text { and }(z, y) \in f \text { for some } z\} \text {. }
$$

Of course, the function $f \circ g$ may well be empty and, in fact, this will be the case precisely when ran $g$ ndom $f=\varnothing$. The empty function is the zero of the semigroup $\mathscr{I}_{F}(X)$ and we will denote it by the symbol 0 . The inverse, in the algebraic sense, of a homeomorphism $f$ in $\mathscr{F}_{F}(X)$ is simply the inverse homeomorphism $f^{-1}$. The idempotents of $\mathscr{I}_{F}(X)$ are the identity maps on closed subsets. The symbol $i_{H}$ will be used to denote the identity map on the closed subset $H$ of $X$. Finally, those homeomorphisms whose domains and ranges each consist precisely of one point play a very important part in our considerations, so we reserve a special notation for them. The symbol $\langle x, y\rangle$ denotes the homeomorphism whose domain is the point $x$ and whose range is the point $y$.

2. Preliminary results on embeddings. This section examines the consequences of having an embedding from $\mathscr{I}_{F}(X)$ into $\mathscr{I}_{F}(Y)$. The information gained here will then be used to prove results about embeddings of $\mathscr{I}_{F}(X)$ into $\mathscr{I}_{F}(Y)$ when the spaces $X$ and $Y$ satisfy certain conditions.

Definition 2.1. Let $\psi$ be an embedding from $\mathscr{I}_{F}(X)$ into $\mathscr{I}_{F}(Y)$. A map $h$ from $X$ into $Y$ is said to be associated with $\psi$ if $h$ is injective, closed with respect to its range and $\psi(f)(h(x))=$ $h(f(x))$ for every $f$ in $\mathscr{I}_{F}(X)$ and $x$ in $\operatorname{dom} f$.

Note that if $f \in \mathscr{I}_{F}(X)$ and $x \in \operatorname{dom} f$ then $h(x) \in \operatorname{dom} \psi(f)$. The next theorem shows that every embedding gives rise to an associated map; we will be interested in situations where the map $h$ turns out to be a homeomorphism.

THEOREM 2.2. Every embedding $\psi$ from $\mathscr{I}_{F}(X)$ into $\mathscr{I}_{F}(Y)$ has an associated map.

Proof. For any $x \in X$, let $R_{x}$ be the subset of $Y$ defined by $i_{R_{x}}=\psi\langle x, x\rangle$ (homomorphic images of idempotents must be idempotents). Let $x, z \in X$. Then $\operatorname{dom}(\psi\langle x, x\rangle)=R_{x}$ and

$$
\begin{aligned}
\operatorname{dom}(\psi\langle x, z\rangle) & =\operatorname{dom}\left((\psi\langle x, z\rangle)^{-1} \circ \psi\langle x, z\rangle\right) \\
& =\operatorname{dom}\left(\psi\left(\langle x, z\rangle^{-1}\right) \circ \psi\langle x, z\rangle\right) \\
& =\operatorname{dom} \psi(\langle z, x\rangle \circ\langle x, z\rangle) \\
& =\operatorname{dom}(\psi\langle x, x\rangle)=R_{x} .
\end{aligned}
$$

Likewise $\operatorname{ran}(\psi\langle x, z\rangle)=R_{z}$. Therefore $\psi\langle x, z\rangle$ is a homeomorphism from $R_{x}$ onto $R_{z}$.

Now we show that if $y \in \operatorname{dom} \psi(0)$, then $y \in \operatorname{dom} \psi(f)$ for every $f \in \mathscr{I}_{F}(X)$ and $\psi(f)(y)=y$. Since 0 is an idempotent, $\psi(0)$ must be an idempotent. Hence if $y \in \operatorname{dom} \psi(0)$, then $\psi(0)(y)=y$. Now for any $f \in \mathscr{I}_{F}(X)$,

$$
\psi(0)=\psi(f \circ 0)=\psi(f) \circ \psi(0) .
$$

This shows that $\operatorname{dom} \psi(0) \subseteq \operatorname{dom} \psi(f)$. If $y \in \operatorname{dom} \psi(0)$ then, by the above,

$$
y=\psi(0)(y)=(\psi(f) \circ \psi(0))(y)=\psi(f)(y) .
$$


Now, in particular, if $x \in X$ then $\operatorname{dom} \psi(0) \subseteq R_{x}$. However, since $0 \neq\langle x, x\rangle$ and $\psi$ is an embedding, we have that $\psi(0) \neq \psi\langle x, x\rangle$. Thus $\operatorname{dom} \psi(0) \subseteq R_{x}$ but dom $\psi(0) \neq R_{x}$.

Now we show that if $x \in \operatorname{dom} f$ then $R_{x} \subseteq \operatorname{dom} \psi(f)$. Since $x \in \operatorname{dom} f$,

Hence $R_{x} \subseteq \operatorname{dom} \psi(f)$.

$$
\begin{aligned}
i_{R_{x}} & =\psi\langle x, x\rangle=\psi\left(i_{\mathrm{dom} f} \circ\langle x, x\rangle\right)=\psi\left(i_{\mathrm{dom} f}\right) \circ i_{R_{x}} \\
& =\psi\left(f^{-1} \circ f\right) \circ i_{R_{x}}=i_{\mathrm{dom} \psi}(f) \circ i_{R_{x}}=i_{\operatorname{dom} \psi}(f) \cap R_{x} .
\end{aligned}
$$

Next we define a map $h$ from $X$ into $Y$ in the following manner. Choose any $x^{\prime} \in X$ and fix it; also choose $y^{\prime} \in R_{x^{\prime}} \mid \operatorname{dom} \psi(0)$ (this is possible by the above results). For $x \in X$ define $h(x)$ by

$$
h(x)=\left(\psi\left\langle x^{\prime}, x\right\rangle\right)\left(y^{\prime}\right) .
$$

Now $h$ is well defined since $\operatorname{dom}\left(\psi\left\langle x^{\prime}, x\right\rangle\right)=R_{x^{\prime}}$ and $y^{\prime} \in R_{x^{\prime}}$ (we remark that $h$ is not necessarily unique).

To prove that $h$ is associated with $\psi$, we will firstly show that $h(x) \in R_{x} \mid \operatorname{dom} \psi(0)$. Clearly $h(x)$ belongs to $R_{x}$ since $\operatorname{ran}\left(\psi\left\langle x^{\prime}, x\right\rangle\right)=R_{x}$. If we also had $h(x) \in \operatorname{dom} \psi(0)$ then

$$
\begin{aligned}
h(x) & =\psi(0)(h(x))=\psi(0)\left(\left(\psi\left\langle x^{\prime}, x\right\rangle\right)\left(y^{\prime}\right)\right) \\
& =\left(\psi\left(0 \circ\left\langle x^{\prime}, x\right\rangle\right)\right)\left(y^{\prime}\right)=\psi(0)\left(y^{\prime}\right)=y^{\prime} .
\end{aligned}
$$

But then $y^{\prime} \in \operatorname{dom} \psi(0)$ which is contrary to the choice of $y^{\prime}$. Hence $h(x) \in R_{x} \mid$ dom $\psi(0)$.

Next we show that $h$ is injective. Suppose $x \neq z$. Then

$$
\psi(0)=\psi(\langle x, x\rangle \circ\langle z, z\rangle)=i_{R_{x}} \circ i_{R_{z}}=i_{R_{x} \cap R_{z}} .
$$

Therefore $R_{x} \cap R_{z}=\operatorname{dom} \psi(0)$. Since $h(x) \in R_{x} \mid \operatorname{dom} \psi(0)$ and $h(z) \in R_{z} \mid$ dom $\psi(0)$, it must be that $h(x) \neq h(z)$.

Now we show that $h$ is closed with respect to its range. Suppose that $F \subseteq X$ and that $F$ is closed. Let $\psi\left(i_{F}\right)=i_{V}$ (homomorphic images of idempotents are idempotents). Then $V$ is closed in $Y$ since $\psi\left(i_{F}\right) \in \mathscr{I}_{F}(Y)$. We will show that $h(F)=V \cap h(X)$. If $x \in F$ then $R_{x} \subseteq V$ (shown previously), and so $h(x) \in V$. Thus $h(F) \subseteq V \cap h(X)$. Conversely, let $h(x) \in V \cap h(X)$. Then $h(x) \in R_{x} \cap V$, and so $h(x) \in \operatorname{dom}\left(i_{V} \circ i_{R_{x}}\right)$. Now $\operatorname{dom}\left(i_{V} \circ i_{R_{x}}\right)=\operatorname{dom} \psi\left(i_{F} \circ\langle x, x\rangle\right)$. Therefore $h(x) \in \operatorname{dom} \psi\left(i_{F} \circ\langle x, x\rangle\right)$. Now $h(x) \notin \operatorname{dom} \psi(0)$ and so $i_{F} \circ\langle x, x\rangle \neq 0$. But then $x \in F$ and so $h(x) \in h(F)$. Hence $h(F)=V \cap h(X)$ and $h$ is closed with respect to its range.

To complete the proof that $h$ is associated with $\psi$ we must show that, if $f \in \mathscr{I}_{F}(X)$ and $x \in \operatorname{dom} f$, then $\psi(f)(h(x))=h(f(x))$. We have shown that $R_{x} \subseteq \operatorname{dom} \psi(f)$ and therefore $h(x) \in \operatorname{dom} \psi(f)$. Now

$$
\psi(f)(h(x))=\left(\psi\left(f \circ\left\langle x^{\prime}, x\right\rangle\right)\right)\left(y^{\prime}\right)=\left(\psi\left\langle x^{\prime}, f(x)\right\rangle\right)\left(y^{\prime}\right)=h(f(x)) .
$$

Thus $h$ is associated with $\psi$.

This last theorem showed that every embedding has at least one associated map. The associated map may not, however, be unique. For example, the map $\psi$ from $\mathscr{I}_{F}(\mathbb{R})$ into $\mathscr{I}_{F}\left(\mathbb{R}^{2}\right)$ defined by

$$
\begin{aligned}
& \operatorname{dom} \psi(f)=\{(x, n): x \in \operatorname{dom} f, \quad n \in \mathbb{N}\} \\
& \psi(f)(x, n)=(f(x), n)
\end{aligned}
$$


is an embedding. For every natural number $n$, define a map $h_{n}$ from $\mathbb{R}$ into $\mathbb{R}^{2}$ by $h_{n}(x)=(x, n)$. It is straightforward to show that $h_{n}$ is associated with $\psi$ for every $n$.

Several results about discrete spaces follow rather easily from the last theorem. Recall that if $X$ is a discrete space, then $\mathscr{I}_{F}(X)$ is the symmetric inverse semigroup on the set $X$.

CoROLlaRY 2.3. Suppose that $X$ is a discrete space and that $\psi$ is an embedding from $\mathscr{I}_{F}(X)$ into $\mathscr{I}_{F}(Y)$. Then $Y$ contains a discrete subspace whose cardinality agrees with that of $X$.

This result follows from the last theorem since the associated map $h$ will in this particular case be a homeomorphism. This means, for example, that if $X$ is discrete and uncountable and $Y$ is second countable, then $\mathscr{I}_{F}(X)$ cannot be embedded into $\mathscr{I}_{F}(Y)$. We now state a result about embeddings when $Y$ is discrete.

COROLlaRY 2.4. Suppose that $X$ is an arbitrary space and that $Y$ is discrete. Then $\mathscr{I}_{F}(X)$ can be embedded into $\mathscr{I}_{F}(Y)$ if and only if $|X| \leqq|Y|$.

Proof. Assume $|X| \leqq|Y|$. Then choose an injective map $\alpha$ from $X$ into $Y$ and, for $f \in \mathscr{I}_{F}(X)$, define a map $\psi$ from $\mathscr{I}_{F}(X)$ into $\mathscr{I}_{F}(Y)$ as follows:

$$
\begin{aligned}
& \operatorname{dom} \psi(f)=\alpha(\operatorname{dom}), \\
& \psi(f)(y)=\alpha(f(x)) \text { for } x \in \operatorname{dom} f, \quad y=\alpha(x) .
\end{aligned}
$$

It is straightforward to show that $\psi$ is an embedding from $\mathscr{I}_{F}(X)$ into $\mathscr{I}_{F}(Y)$.

Conversely, if $\psi$ is an embedding from $\mathscr{I}_{F}(X)$ into $\mathscr{I}_{F}(Y)$, then by Theorem 2.2 there is an injective map $h$ from $X$ into $Y$, and so $|X| \leqq|Y|$.

DEFINITION 2.5. If $\psi$ is an embedding from $\mathscr{I}_{F}(X)$ into $\mathscr{I}_{F}(Y)$ and dom $\psi(f) \subseteq \overline{h(X)}$ for every $f \in \mathscr{I}_{F}(X)$, where $h$ is an associated map of $\psi$, then we say that $\psi$ is a reduced embedding.

DEFINITION 2.6. If $\varphi$ and $\psi$ are two embeddings from $\mathscr{I}_{F}(X)$ into $\mathscr{I}_{F}(Y)$, and if there exists a closed subset $H$ of $Y$ such that $\varphi(f)=\psi(f) \circ i_{H}$ for all $f \in \mathscr{I}_{F}(X)$, we say that $\varphi$ is subordinate to $\psi$.

Every embedding has at least one reduced embedding which is subordinate to it, as may be seen from the next theorem. An embedding may, however, have several reduced embeddings which are subordinate to it. In the example given before, if we define $\varphi_{n}$ (for any natural number $n$ ) from $\mathscr{I}_{F}(\mathbb{R})$ into $\mathscr{I}_{F}\left(\mathbb{R}^{2}\right)$ by

$$
\begin{aligned}
& \operatorname{dom} \varphi_{n}(f)=\{(x, n): x \in \operatorname{dom} f\}, \\
& \varphi_{n}(f)(x, n)=(f(x), n),
\end{aligned}
$$

then $\varphi_{n}$ is a reduced embedding (with associated map $h_{n}$ ) and $\varphi_{n}$ is subordinate to $\psi$.

THEOREM 2.7. For each embedding $\psi$ from $\mathscr{I}_{F}(X)$ into $\mathscr{I}_{F}(Y)$ there is a reduced embedding $\varphi$ which is subordinate to it.

Proof. Suppose $\psi$ is an embedding from $\mathscr{I}_{F}(X)$ into $\mathscr{I}_{F}(Y)$. Let $h$ be an associated map of $\psi$ as defined in Theorem 2.2. For $f \in \mathscr{I}_{F}(X)$ define $\varphi(f)$ by

$$
\varphi(f)=\psi(f) \circ i_{\overline{h(X)}}
$$


where $\overline{h(X)}$ denotes the closure of $h(X)$ in $Y$. Since both $\psi(f)$ and $i_{\overline{h(X)}}$ belong to $\mathscr{I}_{F}(Y)$, it is immediate that $\varphi(f)$ belongs to $\mathscr{I}_{F}(Y)$. Furthermore, if $x \in \operatorname{dom} f$ then $h(x) \in \operatorname{dom} \varphi(f)$ and

$$
\varphi(f)(h(x))=\left(\psi(f) \circ i_{\overline{h(X)}}\right)(h(x))=\psi(f)(h(x))=h(f(x)) .
$$

If, therefore, we can show that $\varphi$ is an embedding from $\mathscr{I}_{F}(X)$ into $\mathscr{I}_{F}(Y)$, we will have that $\varphi$ is a reduced embedding with associated map $h$ and that $\varphi$ is subordinate to $\psi$.

We firstly show that $\varphi(f)$ belongs to $\mathscr{I}_{F}(\overline{h(X)})$. Certainly dom $\varphi(f)$ is a closed subset of $\overline{h(X)}$. Now let $h(x) \in \operatorname{dom} \varphi(f)$. Then $h(x) \in \operatorname{dom} \psi(f)$ and we also have $x \in \operatorname{dom} f$, for otherwise

$$
\psi(0)=\psi(f \circ\langle x, x\rangle)=\psi(f) \circ i_{\boldsymbol{R}_{\boldsymbol{x}}},
$$

and, since $h(x) \in \operatorname{dom} \psi(f) \cap R_{x}$, we would have that $h(x) \in \operatorname{dom} \psi(0)$, contrary to the definition of $h$. Thus $x \in \operatorname{dom} f$ and, since $\varphi(f)(h(x))=h(f(x))$, we have that $\varphi(f)$ maps points of $h(X)$ into $h(X)$. Since $\varphi(f)$ is a homeomorphism this means that $\operatorname{ran} \varphi(f) \subset \overline{h(X)}$ and so $\varphi(f) \in$

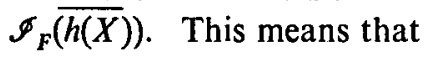

$$
\begin{aligned}
\varphi(f \circ g) & =\psi(f \circ g) \circ i_{\overline{h(X)}}=\psi(f) \circ \psi(g) \circ i_{\overline{h(X)}} \\
& =\psi(f) \circ i_{\overline{h(X)}} \circ \psi(g) \circ i_{\overline{h(X)}}=\varphi(f) \circ \varphi(g),
\end{aligned}
$$

and so $\varphi$ is a homomorphism. Now suppose that $f, g \in \mathscr{I}_{F}(X)$ and that $f \neq g$. If $x \in \operatorname{dom} f \backslash$ dom $g$ then $h(x) \in \operatorname{dom} \varphi(f) \backslash \operatorname{dom} \varphi(g)$ (if $h(x) \in \operatorname{dom} \varphi(g)$ then $x \in \operatorname{dom} g$ ), and so $\varphi(f) \neq \varphi(g)$. If $f(x) \neq g(x)$ and $x \in \operatorname{dom} f \cap \operatorname{dom} g$ then $\varphi(f)(h(x)) \neq \varphi(g)(h(x))$, and so again $\varphi(f) \neq \varphi(g)$. This shows that $\varphi$ is an embedding from $\mathscr{I}_{F}(X)$ into $\mathscr{I}_{F}(Y)$.

The last theorem means that if we have an embedding $\varphi$ from $\mathscr{I}_{F}(X)$ into $\mathscr{I}_{F}(Y)$, then we may assume, without loss of generality, that $\varphi$ is a reduced embedding with an associated map $h$.

3. Main results. This section places restrictions on the topological spaces and then uses the results about reduced embeddings and associated maps to prove several theorems about embeddings.

THEOREM 3.1. Let $\varphi$ be a reduced embedding from $\mathscr{I}_{F}(X)$ into $\mathscr{I}_{F}(Y)$ with associated map $h$, and suppose that both $X$ and $\overline{h(X)}$ are first countable and that $h(X)$ is not discrete in $Y$. Then $h$ is a homeomorphism from $X$ into $Y$.

Proof. Suppose the conditions of the theorem are satisfied. Since $h(X)$ is not discrete there exists $x^{\prime} \in X$ such that $h\left(x^{\prime}\right)$ is a non-isolated point of $h(X)$. By the hypothesis, $h(X)$ is first countable and so there exists a sequence $\left\{h\left(x_{i}\right): i \in \mathbb{N}\right\}$ of distinct points such that $h\left(x_{i}\right) \rightarrow h\left(x^{\prime}\right)$. Now $h$ is an injective closed map and so $h^{-1}$ is continuous. Since $h\left(x_{i}\right) \rightarrow h\left(x^{\prime}\right)$ we have that $h^{-1}\left(h\left(x_{i}\right)\right) \rightarrow h^{-1}\left(h\left(x^{\prime}\right)\right)$ and so $x_{i} \rightarrow x^{\prime}$.

To show that $h$ is a homeomorphism we need only show that $h$ is continuous. Let $z_{i} \rightarrow z$, where $z_{i}, z \in X$. Define a map $f$ as follows:

$$
\begin{aligned}
\operatorname{dom} f & =\left\{x_{i}: i \in \mathbb{N}\right\} \cup\left\{x^{\prime}\right\}, \\
f\left(x_{i}\right) & =z_{i} \text { for } i \in \mathbb{N}, \\
f\left(x^{\prime}\right) & =z
\end{aligned}
$$


Clearly $f \in \mathscr{I}_{F}(X)$. But then $\varphi(f) \in \mathscr{I}_{F}(Y)$ and $h(\operatorname{dom} f) \subseteq \operatorname{dom} \varphi(f)$. This means that $h\left(x_{i}\right) \in \operatorname{dom} \varphi(f)$ for all $i \in \mathbb{N}$ and $h\left(x^{\prime}\right) \in \operatorname{dom} \varphi(f)$. Furthermore,

and

$$
\varphi(f)\left(h\left(x_{i}\right)\right)=h\left(f\left(x_{i}\right)\right)=h\left(z_{i}\right),
$$

$$
\varphi(f)\left(h\left(x^{\prime}\right)\right)=h\left(f\left(x^{\prime}\right)\right)=h(z) .
$$

Now since $\varphi(f)$ is a homeomorphism and $h\left(x_{i}\right) \rightarrow h\left(x^{\prime}\right)$, we have that $h\left(z_{i}\right) \rightarrow h(z)$, and so $h$ is continuous. Hence $h$ is a homeomorphism from $X$ into $Y$.

THEOREM 3.2. Let $\varphi$ be a reduced embedding from $\mathscr{I}_{F}(X)$ into $\mathscr{I}_{F}(Y)$ with associated map $h$. Suppose that $h$ is a homeomorphism. If $\overline{h(X)}$ is first countable, then $|\overline{h(X)} \backslash h(X)| \leqq 1$.

Proof. Let $\varphi$ be a reduced embedding and $h$ a homeomorphism with $\overline{h(X)}$ first countable. Suppose that $|\overline{h(X)} \backslash h(X)| \geqq 2$. Then there exist distinct $z, z^{\prime} \in \overline{h(X)} \backslash h(X)$. Since $\overline{h(X)}$ is first countable, there exist distinct $x_{i}, y_{i} \in X$ for $i \in \mathbb{N}$ where $h\left(x_{i}\right) \rightarrow z$ and $h\left(y_{i}\right) \rightarrow z^{\prime}$. Now since $h$ is a homeomorphism and $z, z^{\prime} \in \overline{h(X)} \backslash h(X)$, we have that $\left\{x_{i}: i \in \mathbb{N}\right\}$ and $\left\{y_{i}: i \in \mathbb{N}\right\}$ are closed discrete subsets of $X$. Define a map $f$ as follows:

$$
\begin{gathered}
\operatorname{dom} f=\left\{x_{i}: i \in \mathbb{N}\right\} \cup\left\{y_{i}: i \in \mathbb{N}\right\}, \\
f\left(x_{i}\right)=x_{2 i} \text { for } i \in \mathbb{N}, \\
f\left(y_{i}\right)=x_{2 i-1} \text { for } i \in \mathbb{N} .
\end{gathered}
$$

Then $f \in \mathscr{I}_{F}(X)$ and so $\varphi(f) \in \mathscr{I}_{F}(Y)$. Since $h$ is an associated map,

and

$$
\varphi(f)\left(h\left(x_{i}\right)\right)=h\left(f\left(x_{i}\right)\right)=h\left(x_{2 i}\right)
$$

$$
\varphi(f)\left(h\left(y_{i}\right)\right)=h\left(f\left(y_{i}\right)\right)=h\left(x_{2 i-1}\right) \text {. }
$$

Now $h\left(x_{i}\right) \rightarrow z$ and $\varphi(f)$ is a homeomorphism, and so $\varphi(f)\left(h\left(x_{i}\right)\right) \rightarrow \varphi(f)(z)$. This means that $h\left(x_{2 i}\right) \rightarrow \varphi(f)(z)$. Likewise $h\left(x_{2 i-1}\right) \rightarrow \varphi(f)\left(z^{\prime}\right)$, since $h\left(y_{l}\right) \rightarrow z^{\prime}$. But $h\left(x_{i}\right) \rightarrow z$, and so $h\left(x_{2 i-1}\right) \rightarrow z$ and $h\left(x_{2 i}\right) \rightarrow z$ also. This means that $\varphi(f)(z)=z=\varphi(f)\left(z^{\prime}\right)$ where $z \neq z^{\prime}$, which is a contradiction. Hence $|\overline{h(X)}| h(X) \mid \leqq 1$.

THEOREM 3.3. Suppose that $h$ is a homeomorphism from $X$ into $Y$, that $\overline{h(X)}$ is compact and first countable, and that $|\overline{h(X)}| h(X) \mid \leqq 1$. Then $\mathscr{I}_{F}(X)$ can be embedded into $\mathscr{I}_{F}(Y)$.

Proof. Without loss of generality we may assume that $X \subseteq Y$. If $\bar{X}=X$ then $\varphi$, defined by $\varphi(f)=f$, will be an embedding from $\mathscr{I}_{F}(X)$ into $\mathscr{I}_{F}(Y)$. Now assume that $|\bar{X}| X \mid=1$ and let $\{z\}=\bar{X} \backslash X$. Define a map $\varphi$ on $\mathscr{I}_{F}(X)$ as follows:

$$
\begin{aligned}
\operatorname{dom} \varphi(f) & =\operatorname{dom} f \cup\{z\}, \\
\varphi(f)(x) & =f(x) \text { if } x \in \operatorname{dom} f \\
\varphi(f)(z) & =z .
\end{aligned}
$$


It is clear that $\varphi(f)$ is an injective map whose domain and range are closed subsets of $Y$. Firstly we show that $\varphi(f)$ is continuous. Let $x_{i} \rightarrow x$ where $x_{i}, x \in \operatorname{dom} \varphi(f)$. If $x \neq z$ then certainly $\varphi(f)\left(x_{i}\right) \rightarrow \varphi(f)(x)$. Now suppose $x=z$. If the sequence $\left\{x_{i}\right\}$ is constant from some point on, then $\varphi(f)\left(x_{i}\right) \rightarrow \varphi(f)(x)$. If not, then let $\left\{x_{i}^{\prime}\right\}$ be the subsequence of $\left\{x_{i}\right\}$ consisting of all points in $\operatorname{dom} f$. Since $\bar{X}$ is compact and first countable, the subsequence $\left\{f\left(x_{i}^{\prime}\right)\right\}$ has an accumulation point. The only choice for this point is $z$, since if $y$ is an accumulation point and $y \in X$, then $y \in \operatorname{ran} f$ and $x_{i} \rightarrow f^{-1}(y)$. But $x_{i} \rightarrow z$. This shows that $\varphi(f)\left(x_{i}\right) \rightarrow z$, and so $\varphi(f)\left(x_{i}\right) \rightarrow \varphi(f)(z)$. This means that $\varphi(f)$ is continuous and, since $\bar{X}$ is compact, we have that $\varphi(f) \in \mathscr{I}_{F}(Y)$.

It is straightforward to show that $\varphi$ is a homomorphism and, since $\varphi$ is clearly injective, this means that $\varphi$ is an embedding from $\mathscr{I}_{F}(X)$ into $\mathscr{I}_{F}(Y)$.

These last results will now be combined to give necessary and sufficient conditions for the existence of an embedding of $\mathscr{I}_{F}(X)$ into $\mathscr{I}_{F}(Y)$ for certain spaces $X$ and $Y$.

THEOREM 3.4. Let $X$ be a first countable space which contains an uncountable number of points. Let $Y$ be any compact metric space. Then $\mathscr{I}_{F}(X)$ can be embedded into $\mathscr{I}_{F}(Y)$ if and only if exactly one of the following two conditions holds:

(i) $X$ is compact and $Y$ contains a copy of $X$;

(ii) $X$ is locally compact but not compact and $Y$ contains a copy of the one-point compactification of $X$.

Proof. If condition (i) or (ii) is satisfied then $\mathscr{I}_{F}(X)$ can be embedded into $\mathscr{I}_{F}(Y)$ by Theorem 3.3.

Suppose, on the other hand, that $\mathscr{I}_{F}(X)$ can be embedded into $\mathscr{I}_{F}(Y)$. By Theorems 2.2 and 2.7 there exists a reduced embedding $\varphi$ of $\mathscr{I}_{F}(X)$ into $\mathscr{I}_{F}(Y)$ with associated map $h$. Since $h$ is injective, $h(X)$ is an uncountable subset of $Y$. Now since $Y$ is a compact metric space, it must be second countable, and hence (see [3, p. 252]) $h(X)$ is not a discrete subspace of $Y$. Thus it follows from Theorem 3.1 that $h$ is a homeomorphism from $X$ into $Y$. Consequently Theorem 3.2 applies and we conclude that $|\overline{h(X)}| h(X) \mid \leqq 1$. If $\overline{h(X)}=h(X)$ then $h(X)$ is compact and so condition (i) is satisfied. If $|\overline{h(X)}| h(X) \mid=1$ then $\overline{h(X)}$ is just the one-point compactification of $h(X)$. This means that $h(X)$ is not compact but is locally compact, and that condition (ii) holds.

For the next results $I^{n}$ will denote the compact $n$-dimensional cube and $S^{n}$ will denote the unit sphere in $\mathbb{R}^{n+1}$ ( $n$ is assumed to be finite). Then, as noted earlier, the one-point compactification of $\mathbb{R}^{n}$ is $S^{n}$. This means that we have the following corollary.

COROLLARY 3.5. $\mathscr{I}_{F}\left(\mathbb{R}^{n}\right)$ can be embedded into $\mathscr{I}_{F}\left(I^{m}\right)$ if and only if $n<m$.

CoRollary 3.6. Let $Y$ be any compact subspace of $\mathbb{R}^{n}$. Then $\mathscr{I}_{F}\left(\mathbb{R}^{n}\right)$ cannot be embedded into $\mathscr{I}_{F}(Y)$. Also, $\mathscr{I}_{F}((0,1))$ cannot be embedded into $\mathscr{I}_{F}([0,1])$.

COROLLARY 3.7. Let $X$ be a locally compact metric space with an uncountable number of points and let $I^{\infty}$ denote the Hilbert cube. Then $\mathscr{I}_{F}(X)$ can be embedded into $\mathscr{I}_{F}\left(I^{\infty}\right)$ if and only if $X$ is separable. 
Proof. Assume that $X$ is a locally compact metric space with an uncountable number of points. Firstly suppose that $\mathscr{I}_{F}(X)$ can be embedded in $\mathscr{I}_{F}\left(I^{\infty}\right)$. Then $I^{\infty}$ contains a copy of $X$ and, since $I^{\infty}$ is separable, $I^{\infty}$ must be second countable, and hence $X$ is second countable and separable.

On the other hand, suppose that $X$ is separable. Now $I^{\infty}$ contains a copy of every separable metric space (see [2, p. 195]), so if $X$ is compact then $I^{\infty}$ contains a copy of $X$ and $\mathscr{I}_{F}(X)$ can be embedded into $\mathscr{I}_{F}\left(I^{\infty}\right)$ by Theorem 3.4. If $X$ is not compact, then, since the one-point compactification of a locally compact separable metric space is metrizable (see [2, p. 247]), we have that $I^{\infty}$ contains a copy of the one-point compactification of $X$, and so by Theorem 3.4 there is an embedding from $\mathscr{I}_{F}(X)$ into $\mathscr{I}_{F}(Y)$.

COROLlaRY 3.8. Let $X$ be a locally compact metric space with an uncountable number of points and let $\mathscr{C}$ denote the Cantor discontinuum. Then $\mathscr{I}_{F}(X)$ can be embedded into $\mathscr{I}_{F}(\mathscr{C})$ if and only if $X$ is separable and 0-dimensional.

Proof. Assume that $X$ satisfies the conditions of the corollary. Suppose that $\mathscr{I}_{F}(X)$ can be embedded into $\mathscr{I}_{F}(\mathscr{C})$. Then $\mathscr{C}$ contains a copy of $X$ and so $X$ must be separable and 0-dimensional.

Now suppose that $X$ is separable and 0 -dimensional. If $X$ is compact, then $\mathscr{C}$ contains a copy of $X$, (see [3, p. 285]) and so, by Theorem 3.4, $\mathscr{I}_{F}(X)$ can be embedded into $\mathscr{I}_{F}(\mathscr{C})$. If $X$ is not compact, then the one-point compactification of $X$ will be topologically contained in $\mathscr{C}$, and so again Theorem 3.4 says that $\mathscr{I}_{F}(X)$ can be embedded into $\mathscr{I}_{F}(\mathscr{C})$.

Now we give an example of compact metric spaces $X$ and $Y$, each with a countable number of points, and an embedding from $\mathscr{I}_{F}(X)$ into $\mathscr{I}_{F}(Y)$ but such that no homeomorphism exists from $X$ into $Y$. This will show the necessity of the requirement in Theorem 3.4 that $X$ be uncountable and also the necessity of the requirement in Theorem 3.1 that $h(X)$ be not discrete.

EXAMPLE. Let $X=H \cup K$ where $H=\{1 / i: i \geqq 3\} \cup\{0\}$ and $K=\{1-1 / i: i \geqq 2\} \cup\{1\}$, and let $Y=\{ \pm 1 / i: i \in \mathbb{N}\} \cup\{0\}$. Assume that $X$ and $Y$ have the inherited topology of the real line. Then both $X$ and $Y$ are compact metric spaces with a countable number of points, but there exists no homeomorphism from $X$ into $Y$. We now define a map $\lambda$ from $X$ into $Y$ as follows:

$$
\begin{aligned}
\lambda(1 / i) & =1 / i \text { for } i \geqq 3, \\
\lambda(1-1 / i) & =-1 / i \text { for } i \geqq 2, \\
\lambda(0) & =-1, \\
\lambda(1) & =1 .
\end{aligned}
$$

For $f \in \mathscr{I}_{F}(X)$ we define $\varphi(f)$ by

$$
\begin{aligned}
\operatorname{dom} \varphi(f) & =\lambda(\operatorname{dom} f) \cup\{0\} \\
\varphi(f)(y) & =\lambda(f(x)) \text { if } y=\lambda(x) \text { and } x \in \operatorname{dom} f, \\
\varphi(f)(0) & =0 .
\end{aligned}
$$

We will sketch the proof that $\varphi$ is an embedding from $\mathscr{I}_{F}(X)$ into $\mathscr{I}_{F}(Y)$. We must firstly show 
that $\varphi(f) \in \mathscr{I}_{F}(Y)$. Clearly $\varphi(f)$ is an injective map whose domain and range are closed subsets of $Y(0 \in \operatorname{dom} \varphi(f) \cap \operatorname{ran} \varphi(f))$. Since $Y$ is compact, we need only show that $\varphi(f)$ is continuous. Hence suppose that $y_{n} \rightarrow y$ where $y_{n}, y \in \operatorname{dom} \varphi(f)$. If the sequence $\left\{y_{n}\right\}$ is constant from some point on, then $\varphi(f)\left(y_{n}\right) \rightarrow \varphi(f)(y)$. Otherwise $y=0$, since 0 is the only limit point of $Y$. We may assume, without loss of generality, that $y_{n} \neq 1$ and $y_{n} \neq-1$ for all $n\left(y_{n} \rightarrow 0\right)$. This means that if $y_{n}=\lambda\left(x_{n}\right)$ for some $n \in \mathbb{N}$ where $x_{n} \in X$, then $x_{n} \neq 0$ and $x_{n} \neq 1$. Now if $\left\{\lambda\left(x_{n}^{\prime}\right)\right\}$ is a subsequence of $\left\{y_{n}\right\}$ and $x_{n}^{\prime} \rightarrow 0$ or $x_{n}^{\prime} \rightarrow 1$, then $f\left(x_{n}^{\prime}\right) \rightarrow 0$ or $f\left(x_{n}^{\prime}\right) \rightarrow 1$, and so $\lambda\left(f\left(x_{n}^{\prime}\right)\right) \rightarrow 0$ $\left(x_{n}^{\prime} \neq 0\right.$ and $x_{n}^{\prime} \neq 1$ for all $n$ ). But this means that $\varphi(f)\left(y_{n}\right) \rightarrow 0$ and hence $\varphi(f)$ is continuous. Thus $\varphi(f) \in \mathscr{I}_{F}(Y)$. Now $\varphi(f)$ is clearly injective and it is straightforward to show that $\varphi$ is a homomorphism. Thus $\varphi$ is an embedding from $\mathscr{I}_{F}(X)$ into $\mathscr{I}_{F}(Y)$.

The results presented in this paper are from the author's doctoral dissertation written at the State University of New York at Buffalo under the direction of K. D. Magill, Jr.

\section{REFERENCES}

1. A. H. Clifford and G. B. Preston, The algebraic theory of semigroups, Math. Surveys of the Amer. Math. Soc. 7 Vol 1 (Providence, R.I., 1961) and Vol. 2 (Providence, R.I., 1967).

2. J. Dugundji, Topology (Allyn and Bacon, Inc., 1967).

3. K. Kuratowski, Topology Vol. I (Academic Press, 1966).

4. G. B. Preston, Representations of inverse semi-groups, J. London Math. Soc. 29 (1954), $411-419$.

5. V. V. Vagner, Generalized Groups, Dokl. Akad. Nauk SSSR (N.S.) 84 (1952), 1119-1122.

State University of New York at Buffalo

AMHERST, NEW YORK 14226

U.S.A.

PRINTED IN QREAT BRITAIN BY ROBERT MAOLEHOSE AND CO. LTD PRINTERS TO THE UNIVERSITY OP GLASGOW 